\title{
X-ray Absorption Fine Structure Investigation of the Low Temperature Grown ZnCoO Films
}

\author{
A. Wolska ${ }^{a, *}$, M.T. Klepka ${ }^{a}$, B.S. Witkowski ${ }^{a}$, M.I. Łukasiewicz ${ }^{a}$, \\ E. Guziewicz ${ }^{a}$, M. Godlewski ${ }^{a, b}$
}

${ }^{a}$ Institute of Physics PAS, al. Lotników 32/46, 02-668, Warsaw, Poland

${ }^{b}$ Dept. Math. and Natural Sciences College of Science, Cardinal S. Wyszyński Univ., Warsaw, Poland

$\mathrm{ZnO}$ based diluted magnetic semiconductors are intensively investigated for possible spintronic applications. In the present work we investigate the $\mathrm{ZnCoO}$ layers grown at low temperature by atomic layer deposition. The local atomic structure of a series of layers with different Co concentration is investigated by the X-ray absorption fine structure measurements. Two groups of $\mathrm{ZnCoO}$ layers are investigated - the ones with an uniform Co distribution and highly nonuniform films. For uniform samples we observe that a majority of Co atoms is built into the $\mathrm{ZnO}$ matrix substituting the $\mathrm{Zn}$ atoms. In contrast, for the nonuniform samples, metallic Co inclusions are also observed. These results are in strong correlation with the magnetic properties of the films studied separately. Samples with the uniform Co distribution (Co substitutes $\mathrm{Zn}$ in $\mathrm{ZnO}$ ) are paramagnetic, whereas the nonuniform ones show a ferromagnetic response.

PACS: 78.70.Dm, 75.50.Pp, 68.55.-a

\section{Introduction}

Diluted magnetic semiconductors of two wide band gap materials ( $\mathrm{GaN}$ and $\mathrm{ZnO}$ ) are intensively investigated for possible spintronic applications. Among the ZnO-based compounds, the thin films of $\mathrm{ZnCoO}$ are the most studied, which is due to the observation of a room temperature (RT) ferromagnetism (FM) [1]. However, it turned out soon that the reported FM is not due to "the bulk properties" of studied samples, but due to a presence of foreign phases and Co metal inclusions. This we investigated in details in our recent publication [2]. Similar situation we also found for $\mathrm{ZnMnO}$ layers, studied by us separately [3-4]. The most crucial question to answer is thus how a given transition metal enters lattice of investigated materials and, if inclusions of foreign phases or metal accumulations are observed, what is the relation between their presence and magnetic properties of investigated films.

In the present work we answer these questions investigating lattice sites and local environment of Co ions in two groups of $\mathrm{ZnCoO}$ layers. These samples were selected based on the results of our recent investigations. Samples with a uniform Co distribution (grown at a reduced temperature) were paramagnetic, whereas the ones grown at an increased temperature were less uniform. For the latter samples we observed a FM response even at RT. We employ here two techniques - the X-ray absorption near edge structure (XANES) and extended X-ray absorption fine structure (EXAFS), which are highly sensitive to local environments in investigated samples.

\section{Sample preparation}

The $\mathrm{ZnCoO}$ layers were grown by the atomic layer deposition (ALD) method. Information on their growth

\footnotetext{
* corresponding author; e-mail: wolska@ifpan.edu.pl
}

temperature $\left(160{ }^{\circ} \mathrm{C}, 200^{\circ} \mathrm{C}\right.$ and $\left.300^{\circ} \mathrm{C}\right)$, layers thickness, and Co fraction is given in Table I. Paramagnetic films are marked as group A, whereas the ferromagnetic ones as group B.

TABLE I

List of the investigated $\mathrm{ZnCoO}$ layers grown at low temperature by the ALD method. Paramagnetic films are marked as the group A, whereas the ferromagnetic ones as the group B.

\begin{tabular}{l|c|c|c|c}
\hline \hline Name & $\begin{array}{l}\text { growth tem- } \\
\text { perature [ }\left[{ }^{\circ} \mathrm{C}\right]\end{array}$ & $\begin{array}{l}\text { layer thick- } \\
\text { ness [nm] }\end{array}$ & Co [\%] & group \\
\hline F175 & 160 & 960 & 2.8 & A \\
F254 & 160 & 70 & 13.5 & A \\
F268 & 200 & 400 & 3.4 & B \\
F274 & 200 & 370 & 4.5 & A \\
F307 & 200 & 240 & 6.5 & B \\
F328 & 200 & 50 & 19.5 & B \\
F279 & 300 & 1400 & 3.5 & A \\
F213 & 300 & 130 & 5.2 & A
\end{tabular}

Further details on the growth conditions, precursors used, lengths of the ALD pulses, evaluation of Co content, etc can be found in the reference [2]. Here we only underline that in addition to a growth temperature the second critical parameter turned out to be a ratio of $\mathrm{ZnO}$ to $\mathrm{CoO}$ pulses in the ALD process. Both these factors affect uniformity of investigated $\mathrm{ZnCoO}$ layers.

\section{Experiment}

The X-ray absorption fine structure (XAFS) technique is element selective which guarantees that the local atomic neighborhood of the Co atoms can be studied unambiguously. The XAFS measurements at the $\mathrm{K}$ edge of Co were performed at DESY - HASYLAB (Cemo station) at liquid nitrogen temperature. The thin layers 
were measured in a fluorescence mode using a 7-element silicon fluorescence detector. The reference samples of metallic Co and cobalt oxides were measured in a transmission mode.

The $a b$ initio calculations of the XANES spectra using the FEFF 8.4 code [5] were conducted during the XANES analysis. EXAFS analysis was carried out using IFEFFIT data analysis package with Athena and Artemis programs [6].

\section{Results and discussion \\ 4.1 XANES analysis}

The XANES spectra are sensitive to the local structure around a selected element. They can be treated as a fingerprint of the formed chemical bonds. XANES spectra of the investigated layers are shown in Fig. 1.

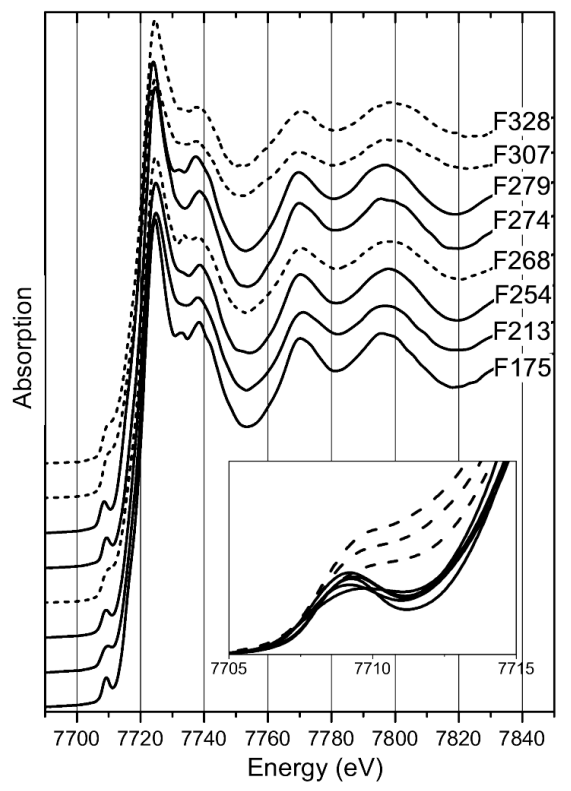

Fig. 1. Normalized Co K-edge XANES spectra of the thin layer samples. The full lines correspond to samples from group A, dashed lines from group B. Spectra are shifted vertically for clarity. The inset shows the preedge region of the same samples.

Their shapes are similar to each other, however, some distinct differences among spectra for paramagnetic (group A) and ferromagnetic (group B) samples are observed. The main difference is the presence of a pre-peak around $7709.3 \mathrm{eV}$. For the group A the pre-peak is clear and pronounced, while for the group B it is very weak, looking like a bump (see the inset in Fig. 1). This pre-peak is associated with the transition of Co 1 s electron to $4 p-$ $3 \mathrm{~d}$ hybridized states indicating the tetrahedral symmetry [7-8]. Its presence in the spectra of group A suggests that in this case the Co atoms are surrounded by four neighboring atoms.

The shapes and the positions of the next four peaks: around $7724.6 \mathrm{eV}, 7738.4 \mathrm{eV}, 7770.4 \mathrm{eV}$ and $7797.3 \mathrm{eV}$ are similar for all investigated spectra. However, for the group B the peaks' amplitudes are weaker than for the group A, just like it was for the pre-peak. It suggests that even though the majority of the Co atoms forms the same compound as in the samples from the group A, there also exists another phase, smoothing the spectral features. The EXAFS oscillations presented in Fig. 2 confirm differences between the groups.

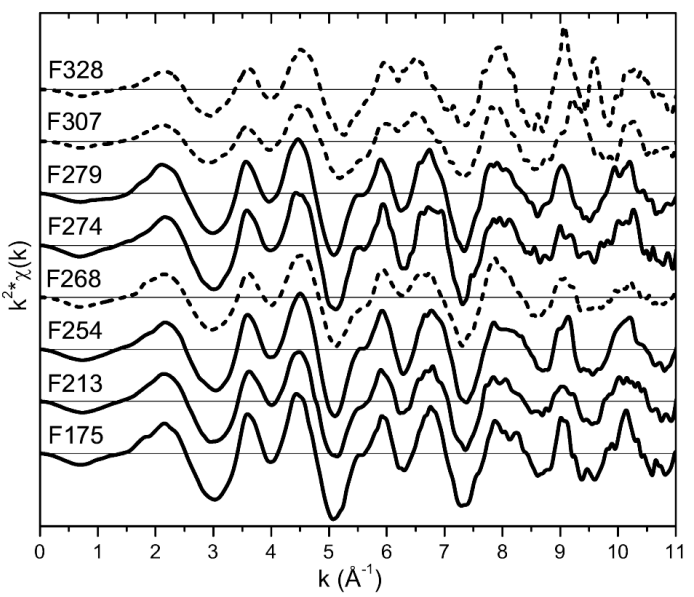

Fig. 2. The EXAFS oscillations at the Co K-edge. The full lines correspond to samples from group A, dashed lines from group B. Spectra are shifted vertically for clarity.

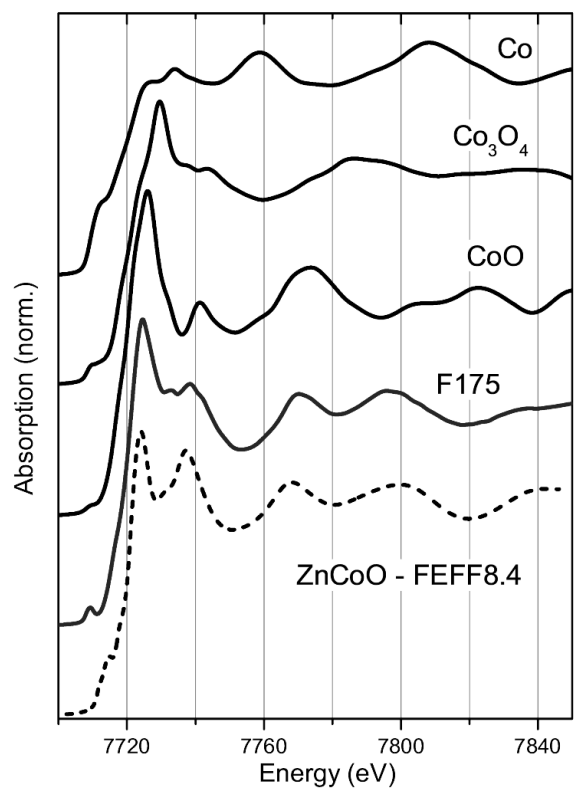

Fig. 3. Normalized XANES spectra of one of the samples (F175) and the Co and Co oxides references. The $\mathrm{ZnCoO}$ spectrum (dashed line) represents calculations for the model where Co substitutes $\mathrm{Zn}$ in $\mathrm{ZnO}$. Spectra are shifted vertically for clarity.

There are several possibilities while considering possible Co containing phases. The Co atoms can be built 
in the $\mathrm{ZnO}$ matrix ( $\mathrm{ZnCoO}$ compound), form metallic Co clusters or Co oxide inclusions. In Fig. 3 the reference $\mathrm{Co}, \mathrm{Co}_{3} \mathrm{O}_{4}$ and $\mathrm{CoO}$ spectra are presented. Their shapes differ considerably from the F175 spectrum shown here for the comparison. This is in agreement with the conclusion drawn from the presence of the pre-peak. In metallic Co the first coordination sphere consists of 12 Co atoms at the distance $2.51 \AA$, for $\mathrm{CoO}-6$ oxygen atoms at $2.13 \AA$ and for $\mathrm{Co}_{3} \mathrm{O}_{4}-5.3$ oxygen atoms at the average distance $1.95 \AA$. The tetrahedral symmetry can be only achieved in the case of the Co atoms located in a substitutional position in the $\mathrm{ZnO}$ structure.

In order to confirm this possibility, we conducted the calculations of the $\mathrm{ZnCoO}$ spectrum. A cluster with a $10 \AA$ radius was first created using the known crystallographic data for the $\mathrm{ZnO}$ structure [9]. Then, the central Zn atom was substituted by a Co atom and the XANES spectrum was calculated using the XANES, SCF (SelfConsistent Field) and FMS (Full Multiple Scattering) cards. The Hedin-Lundqvist potential was chosen. The resulting spectrum is presented in Fig. 3. Its shape is close to the spectrum of the F175 sample, indicating that in the samples from the group A, the Co atoms are built into the $\mathrm{ZnO}$ structure forming the $\mathrm{ZnCoO}$ alloy.

\subsection{EXAFS analysis}

The EXAFS analysis provides quantitative information about the distribution of the atoms around the selected element. The XANES analysis led to the conclusion that in the case of the samples from the group $\mathrm{A} \mathrm{Co}$ atoms are built into the $\mathrm{ZnO}$ structure. Thus, this model was considered as the first in the analysis of the EXAFS data. The Fourier transformed (FT) EXAFS spectra and their fits for the samples from the group A are presented in Fig. 4. Four clear coordination spheres up to $5.7 \AA$ can be distinguished indicating a high crystalline quality of the studied layers. The fitting was performed in the $R$ range from 1 to $3.5 \AA$. The value of the amplitude reduction factor $\left(\mathrm{S}_{0}^{2}\right)$ was estimated as equal to 0.8 from fitting of the first coordination sphere of the F175 sample. This value was then used in all performed fits. The fitting parameters are collected in Table II. The $R$-factor measures the absolute misfit between the data and theory and is used for the determination of the fit quality. In case of the fit for the $\mathrm{CoO}$ reference (not shown here) the value of $R$-factor was equal to 0.025 . For all of the presented fits, the $R$-factor is close to that value and lower than 0.05 , which means that the fits are quite exact.

According to the crystallographic data for $\mathrm{ZnO}$ (P6 $3 / \mathrm{mmc}$ space group) [9], the first coordination sphere around $\mathrm{Zn}$ consists of 4 oxygen atoms, one at the distance of $1.80 \AA$ and three at $2.04 \AA$. The second sphere consists of two subshells, both of them with six $\mathrm{Zn}$ atoms at 3.21 $\AA$ and $3.25 \AA$, respectively. The substitution of $\mathrm{Zn}$ atoms by Co atoms causes a change in the arrangement of the first sphere, resulting in tetrahedral environment, with all 4 oxygen atoms located at the same distance equal to $1.97 \AA$. Importantly, the $\mathrm{Co}-\mathrm{Zn}$ distance found from the

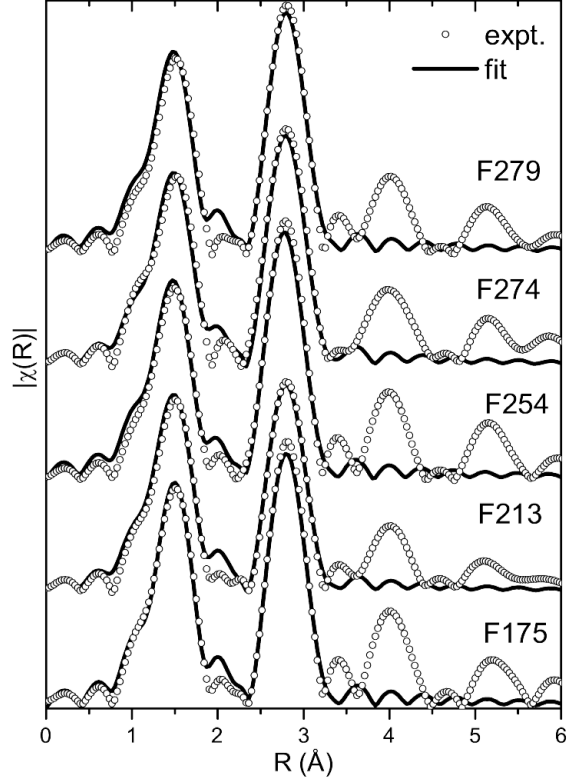

Fig. 4. Results of the fitting (in $\mathrm{R}$ space) of the EXAFS spectra of the samples from group A. Spectra are shifted vertically for clarity. Empty circles represent experiment, full lines - obtained fits.

TABLE II

The fitting parameters of the EXAFS spectra of the samples from group A. $R$ - a distance to the central atom, $\sigma^{2}$ - EXAFS Debye-Waller factor, $R$-factor indicates quality of the fit. The $S_{0}^{2}$ parameter is set to 0.8 . The numbers of neighbors $(N)$ were kept according to the crystallographic data: $N_{\mathrm{O}}=4, N_{\mathrm{Zn} 1}=6, N_{\mathrm{Zn} 2}=6$

\begin{tabular}{l|l|l|l|l|l}
\hline \hline & \multicolumn{1}{|c|}{ F175 } & \multicolumn{1}{c|}{ F213 } & \multicolumn{1}{c}{ F254 } & \multicolumn{1}{c}{ F274 } & \multicolumn{1}{c}{ F279 } \\
\hline$R_{\mathrm{O}}[\AA]$ & $1.97(1)$ & $1.97(1)$ & $1.97(1)$ & $1.97(1)$ & $1.97(1)$ \\
$\sigma_{\mathrm{O}}^{2}\left[\AA^{2}\right]$ & $0.003(2)$ & $0.005(1)$ & $0.005(2)$ & $0.005(2)$ & $0.004(2)$ \\
$R_{\mathrm{Zn} 1}[\AA]$ & $3.20(1)$ & $3.21(1)$ & $3.19(1)$ & $3.20(1)$ & $3.21(1)$ \\
$R_{\mathrm{Zn} 2}[\AA]$ & $3.24(1)$ & $3.25(1)$ & $3.23(1)$ & $3.24(1)$ & $3.25(1)$ \\
$\sigma_{\mathrm{Zn}}^{2}\left[\AA^{2}\right]$ & $0.008(1)$ & $0.010(1)$ & $0.008(1)$ & $0.009(1)$ & $0.008(1)$ \\
$R$-factor & 0.027 & 0.026 & 0.034 & 0.028 & 0.038
\end{tabular}

fitting is close to the $\mathrm{Zn}-\mathrm{Zn}$ distance in the $\mathrm{ZnO}$ structure. It means that Co atoms disturb only the first shell, and that the disturbance does not spread through the lattice.

Analysis of the XANES data for the samples from the group $\mathrm{B}$ indicates the presence of two phases in the studied layers. In addition to the dominant $\mathrm{ZnCoO}$ phase an unknown phase influences the data. Indeed, the FT EXAFS spectra for this group clearly show an additional peak between the two peaks observed for the group A. The localization of this peak is consistent with the one expected for the first coordination sphere of metallic Co. It means that a part of the Co atoms is built into the $\mathrm{ZnO}$ structure forming the $\mathrm{ZnCoO}$ alloy, while remaining $\mathrm{Co}$ atoms form metallic inclusions. In Fig. 5 the inputs from both phases are shown for the F328 sample. These inputs are calculated using the parameters from the fit. Experi- 


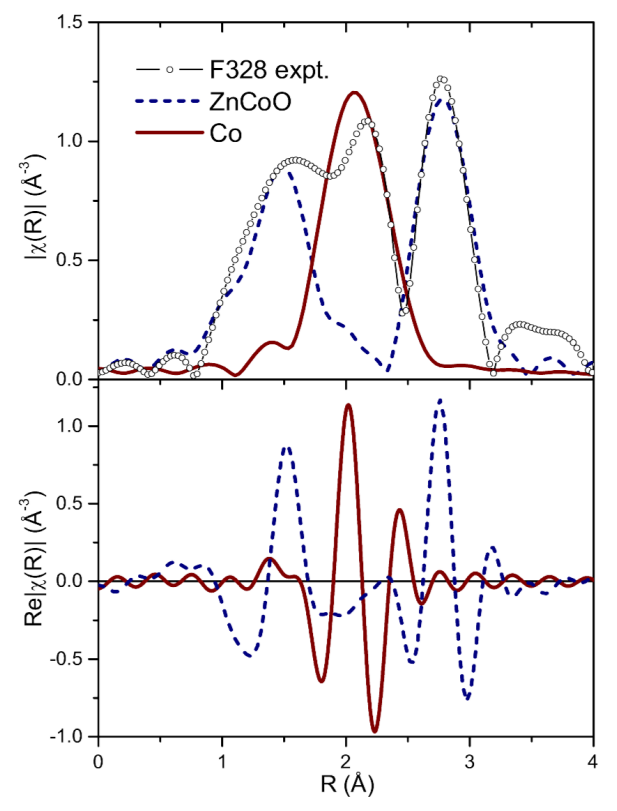

Fig. 5. The magnitudes (upper curves) and real part of Fourier transforms (lower curves) for $\mathrm{ZnCoO}$ (dashed lines) and Co (full lines) contributions obtained from the fitting of the F328 sample. The magnitude of the experimental spectrum is also shown (empty circles).

mental data are perfectly reproduced, when we assumed coexistence of two phases mentioned above.

The FT EXAFS spectra for the samples from group $\mathrm{B}$ and their fits are presented in Fig. 6. We found that the presence of the additional phase in the layers influences not only the shape of the closest peaks. Also the peaks corresponding to the third and fourth coordination sphere are no longer clearly recognizable in the spectra for the F307 and F328 samples.

The fitting was performed for $R$ in the range $\sim 1$ to $3.6 \AA$. The $S_{0}^{2}$ parameter was set to 0.8 as in the previous case. Additionally, the $\mathrm{x}$ parameter was introduced representing the percentage of the Co atoms in metal inclusions. The fitting parameters are collected in Table III.

For all considered samples the $\mathrm{Co}-\mathrm{Zn}$ distances in $\mathrm{ZnO}$ and $\mathrm{Co}-\mathrm{Co}$ distances in Co phase are, within the error, consistent with the crystallographic data. Unfortunately, the percent of the Co atoms forming the metal inclusions cannot be determined with a high accuracy. Its value is roughly between 20 and $30 \%$ of all Co atoms present in the sample.

In case of the F268 sample the $\mathrm{x}$ value is only slightly smaller than for the F307 and F328 samples. On the other hand the EXAFS Debye-Waller factor $\left(\sigma^{2}\right)$ is considerably higher for the inclusions formed in this sample than for the ones in the F328 and F307 samples.

The $\sigma^{2}$ parameter consists of two terms describing the attenuation of the EXAFS signal due to the thermal and structural disorder. The part related to the thermal disorder can be minimized by performing measurements at

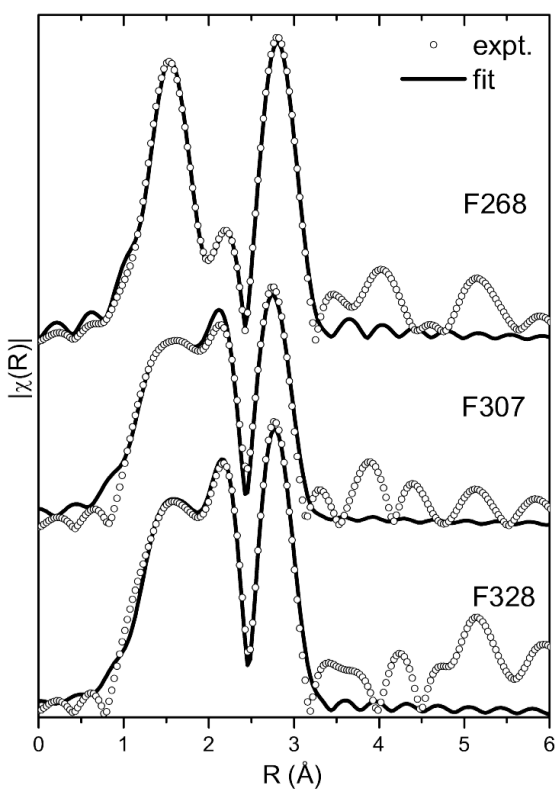

Fig. 6. Results of the fitting (in R space) of the EXAFS spectra of the samples from group B. Spectra are shifted vertically for clarity. Empty circles represent experiment, full lines - obtained fits.

TABLE III

The fitting parameters of the EXAFS spectra of the samples from group B. $R$ - a distance to the central atom, $\sigma^{2}$ - EXAFS Debye-Waller factor, $R$-factor indicates quality of the fit. The $\mathrm{S}_{0}^{2}$ parameter is set to 0.8 . The numbers of neighbors $(\mathrm{N})$ were kept according to the crystallographic data: $N_{\mathrm{O}}=4, N_{\mathrm{Zn} 1}=6, N_{\mathrm{Zn} 2}=6, N_{\mathrm{Co}}=12$

\begin{tabular}{l|l|l|l}
\hline \hline & \multicolumn{1}{|c|}{ F328 } & \multicolumn{1}{|c}{ F307 } & \multicolumn{1}{|c}{ F268 } \\
\hline$R_{\mathrm{O}}[\AA]$ & $1.98(2)$ & $1.96(2)$ & $1.99(1)$ \\
$\sigma_{\mathrm{O}}^{2}\left[\AA^{2}\right]$ & $0.007(3)$ & $0.009(4)$ & $0.004(3)$ \\
$R_{\mathrm{Zn} 1}[\AA]$ & $3.19(2)$ & $3.18(3)$ & $3.21(2)$ \\
$R_{\mathrm{Zn} 2}[\AA]$ & $3.23(2)$ & $3.22(3)$ & $3.25(2)$ \\
$\sigma_{\mathrm{Zn}}^{2}\left[\AA^{2}\right]$ & $0.009(2)$ & $0.011(2)$ & $0.008(2)$ \\
$\mathrm{x}[\%]$ & $27(8)$ & $25(8)$ & $24(10)$ \\
$R_{\mathrm{Co}}[\AA]$ & $2.49(2)$ & $2.48(3)$ & $2.46(6)$ \\
$\sigma_{\text {Co }}^{2}\left[\AA^{2}\right]$ & $0.005(3)$ & $0.006(3)$ & $0.011(5)$ \\
$R$-factor & 0.021 & 0.028 & 0.015
\end{tabular}

low temperatures, as it was in this case. Therefore, we assume that only the structural part, responsible for atomic displacements or presence of vacancies, plays a significant role here. We thus expect that for the F268 sample the metallic Co inclusions are very small or highly defected, which lower their influence on the spectrum. This conclusion is consistent with the fact that in the FT EXAFS spectrum the peaks corresponding to the third and fourth shell in $\mathrm{ZnCoO}$ can be distinguished, as it was for the samples from group A.

\section{Conclusions}

A local atomic structure around the Co atoms in the $\mathrm{ZnCoO}$ samples grown by the ALD method was investi- 
gated using XANES and EXAFS techniques. The performed analysis reveals that in the paramagnetic samples from group $\mathrm{A}$ the Co atoms are built into the $\mathrm{ZnO}$ structure substituting the $\mathrm{Zn}$ atoms and forming a uniform $\mathrm{ZnCoO}$ layer. These samples do not exhibit ferromagnetic properties, as studied separately.

The rest of the samples (group B) shows a ferromagnetic response. It is correlated with the local surrounding of the Co atoms. Even though the majority of Co atoms is built into $\mathrm{Zn}$ sites forming the $\mathrm{ZnCoO}$ alloys, about 20 $30 \%$ of Co atoms forms metallic Co inclusions. From the separate study we conclude that the presence of this Co phase results in the ferromagnetic response of the layers $[2]$.

The F328 and F307 samples show a strong ferromagnetic response while in the case of the F268 sample such a response is much weaker. This behavior can be explained by the results of the EXAFS analysis. The $\sigma_{\text {Co }}^{2}$ parameter indicates that for the samples with a strong ferromagnetism the Co inclusions are rather large with a good quality crystalline structure. In the case of the F268 sample the $\sigma_{\text {Co }}^{2}$ parameter is considerably higher indicating that the Co clusters are very small or highly defected. It implies that the ferromagnetic properties depend on the quality of the Co inclusions.

\section{Acknowledgments}

This work was supported by the European Union within European Regional Development Fund, through grant Innovative Economy (POIG.01.01.02-00-008/08).
The research leading to these results has received funding from the European Community's Seventh Framework Programme (FP7/2007-2013) under Grant agreement no. 226716.

\section{References}

[1] K. Sato, H. Katayama-Yoshida, Jpn. J. Appl. Phys., Part 2 40, L334 (2001).

[2] M. Godlewski, E. Guziewicz, M.I. Łukasiewicz, I.A. Kowalik, M. Sawicki, B.S. Witkowski, R. Jakieła, W. Lisowski, J.W. Sobczak, M. Krawczyk, Physica Status Solidi (b) 248, 1596 (2011).

[3] A. Wójcik, K. Kopalko, M. Godlewski, E. Guziewicz, R. Jakieła, R. Minikayev, W. Paszkowicz, Appl. Phys. Lett. 89, 051907 (2006).

[4] A. Wójcik, M. Godlewski, E. Guziewicz, K. Kopalko, R. Jakieła, M. Kiecana, M. Sawicki, M. Guziewicz, M. Putkonen, L. Niinistö, Y. Dumont, N. Kelner, Appl. Phys. Lett. 90, 082502 (2007).

[5] A.L. Ankudinov, B. Ravel, J.J. Rehr, S.D. Conradson, Phys. Rev. B 58, 7565 (1998).

[6] B. Ravel, M. Newville, J. Synchrotron Rad. 12, 537 (2005).

[7] Z. Sun, W. Yan, G. Zhang, H. Oyanagi, Z. Wu, Q. Liu, W. Wu, T. Shi, Z. Pan, P. Xu, S. Wei, Phys. Rev. B 77, 245208 (2008).

[8] R. Djenadic, G. Akgul, K. Attenkofer, M. Winterer, J. Phys. Chem. C 114, 9207 (2010).

[9] R.W.G. Wyckoff Crystal Structures I. Second edition, Interscience Publishers, New York 1963. 